Rev. Int. Contam. Ambie. 32 (3) 303-313, 2016

DOI: 10.20937/RICA.2016.32.03.05

\title{
EVALUACIÓN Y CARACTERIZACIÓN DE GRASAS Y ACEITES RESIDUALES DE COCINA PARA LA PRODUCCIÓN DE BIODIÉSEL: UN CASO DE ESTUDIO
}

\author{
Veymar Guadalupe TACIAS PASCACIO ${ }^{1}$, Arnulfo ROSALES QUINTERO ${ }^{2 *}$ y \\ Beatriz TORRESTIANA SÁNCHEZ ${ }^{1}$
}

${ }^{1}$ Unidad de Investigación y Desarrollo en Alimentos, Instituto Tecnológico de Veracruz. Avenida Miguel Ángel de Quevedo 2779. Colonia Formando Hogar, Veracruz, Veracruz, México, C.P. 91897

${ }^{2}$ Instituto Tecnológico de Tuxtla Gutiérrez. Carretera Panamericana km. 1080, Apartado Postal 599, Tuxtla Gutiérrez, Chiapas C.P. 29000

*Autor para correspondencia: btorrest@itver.edu.mx

(Recibido mayo 2015; aceptado noviembre 2015)

Palabras clave: contaminación, residuos, aprovechamiento, biocombustibles, diésel

\section{RESUMEN}

Se evaluó de forma sistemática la cantidad de grasas y aceites (GAR) generada por la industria restaurantera en la ciudad de Tuxtla Gutiérrez, Chiapas. Los resultados indicaron que en la ciudad se producen en promedio $174 \mathrm{t}$ de GAR por año provenientes de ocho tipos de restaurantes en la siguiente proporción: $41 \%$ restaurantes formales, $24 \%$ comida rápida, $8 \%$ taquerías, $7 \%$ antojitos, $6 \%$ cocinas económicas, $6 \%$ bares, $4 \%$ gorditas y $4 \%$ rosticerías. Además, muestras representativas de GAR provenientes de cada tipo de restaurante fueron caracterizadas para la producción de biodiésel en términos de viscosidad, estabilidad oxidativa, contenido de ácidos grasos libres (AGL), índice de acidez (IA), índice de saponificación, contenido de humedad y composición de ácidos grasos. El análisis fisico y químico indicó que todas, excepto las GAR generadas por restaurantes de comida rápida, cumplen con los niveles de AGL e IA, recomendados para la transesterificación alcalina. Considerando la población de la ciudad, se estimó un promedio de producción de GAR de 0.31 t/año por cada 1000 habitantes. Un análisis basado en estas cifras sugiere que en México se podrían obtener $34.9 \mathrm{kt}$ de biodiésel/año y evitar la emisión de $92 \mathrm{kt} \mathrm{de} \mathrm{CO}_{2} /$ año, reduciendo así la contaminación en el país.

Key words: pollution, waste, exploitation, biofuel, diesel

\begin{abstract}
The waste cooking oils and fats (WCOF) from the catering industry were systematically evaluated in Tuxtla Gutierrez, Chiapas. Results pointed out that the eight restaurant types found in the city produced $174 \mathrm{t}$ of WCOF/year in the following proportion: $41 \%$ formal restaurant, $24 \%$ fast food, $8 \%$ taquerias, $7 \%$ antojitos, $6 \%$ soup kitchens, $6 \%$ bars, $4 \%$ gorditas and $4 \%$ roast chicken shops. In addition, representative samples of WCOF coming from every restaurant type were characterized for biodiesel production, in terms of viscosity, oxidative stability, free fatty acid content, acid value, saponification number, moisture content and fatty acid composition.
\end{abstract}


The physicochemical analysis suggests that all the WCOF produced in the city, except that coming from the fast food cooking, met the recommended free fatty acid levels and the acidity values for alkaline transesterification. Based on the population of the city an average production of $0.31 \mathrm{t} /$ year of WCOF per 1000 inhabitants was estimated. Projecting this figure to the whole country of Mexico, $34.9 \mathrm{kt}$ of this fuel would be obtained per year, which would avoid the emission of $92 \mathrm{kt} / \mathrm{year}$ of $\mathrm{CO}_{2}$ and contribute to reduce pollution in the country.

\section{INTRODUCCIÓN}

El aumento en la población mundial, la búsqueda de la comodidad y las actividades diarias, son factores que contribuyen al incremento en el consumo de energía y a la generación de residuos. Los problemas ambientales (emisión de gases de efecto invernadero y la lluvia ácida) derivados de las actividades humanas, además de los problemas económicos asociados con el incremento en el precio y la escases del petróleo, han estimulado la búsqueda de combustibles renovables con el objetivo de reducir el consumo o incluso sustituir completamente a los combustibles derivados del petróleo (Mohibbe et al. 2005, Canesin et al. 2014).

El biodiésel es un combustible alternativo al diésel de petróleo, es un producto biodegradable, no tóxico y menos perjudicial para el ambiente en comparación con el diésel de petróleo. Este combustible es una mezcla de metil ésteres de ácidos grasos (FAME, por sus siglas en inglés), obtenidos de recursos renovables, como los aceites vegetales y las grasas animales, con bajo valor comercial (Dias et al. 2009, Ibeto et al. 2011). El biodiésel es obtenido por transesterificación de triglicéridos, constituyentes principales de los aceites vegetales y grasas animales, con un alcohol de cadena corta (metanol, principalmente), en presencia de un catalizador adecuado. Los catalizadores alcalinos como el hidróxido de potasio o de sodio y los metóxidos de potasio y sodio, son los catalizadores más comúnmente utilizados en la producción de biodiésel (Predojević 2008). La transesterificación consiste en tres reacciones consecutivas reversibles donde los triglicéridos son convertidos a diglicéridos, los diglicéridos a monoglicéridos y estos a glicerol (subproducto principal de la reacción). En cada etapa se usan tres moléculas de alcohol por molécula de triglicérido. Estequiométricamente se producen tres moles de éster por cada mol de triglicérido (Sharma y Singh 2008) como se indica de forma resumida en la reacción 1:

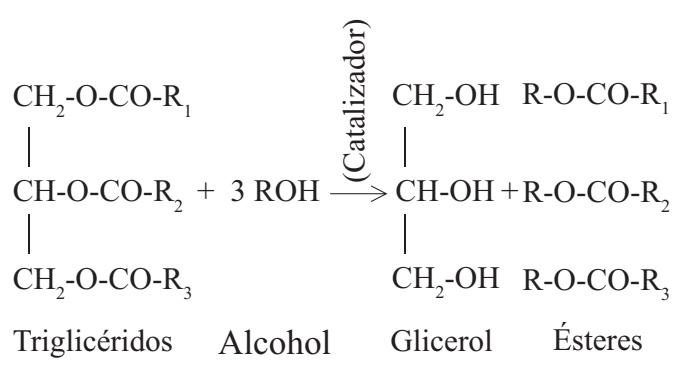

La principal desventaja de la catálisis alcalina es su alta sensibilidad a la pureza de los reactivos, así como al contenido de ácidos grasos libres y agua. El contenido de ácidos grasos libres no debe exceder ciertos límites, debido a que pueden promoverse reacciones indeseadas de saponificación (reacción 2). Esto reduce la eficiencia del catalizador y el rendimiento de ésteres (biodiésel). Además dificulta los procesos de separación de ésteres y glicerol (Cao et al. 2006, Huang et al. 2010).

$$
\begin{array}{ccc}
\mathrm{R}-\mathrm{COOH}+\mathrm{NaOH} \text { ó } \mathrm{KOH} \rightarrow & \mathrm{RCOONa}^{+} \\
\mathrm{AGL} & \begin{array}{c}
\text { Catalizador } \\
\text { alcalino }
\end{array} & \text { ó } \mathrm{RCOOK}^{+}+\mathrm{H}_{2} \mathrm{O} \\
& \text { Jabón }
\end{array}
$$

El biodiésel obtenido a partir de aceites vegetales es una alternativa para reemplazar parte del petróleo demandado. Sin embargo, las grandes cantidades de aceite comestible requeridas para la generación de energía pueden conducir a una crisis en el suministro de alimentos y a un desequilibrio económico (Canesin et al. 2014). Por otro lado, la generación de aceites residuales se está convirtiendo en un problema creciente en todo el mundo (Felizardo et al. 2006, Kulkarni y Dalai 2006, Chhetri et al. 2008, Predojević 2008). El manejo de las grasas y aceites residuales (GAR) es un reto importante, debido a los problemas relacionados con su eliminación y a su posible contribución a la contaminación del agua y el suelo (Chhetri et al. 2008). Con base en estimaciones reportadas en siete países, en la Unión Europea se producen alrededor de 0.4 Megatoneladas (Mt) de GAR, principalmente provenientes de la industria restaurantera, mientras que en Irlanda, la cantidad 
de aceite de cocina residual reciclado fue de $9381 \mathrm{t}$ en el año 2006 (Singh et al. 2010). Hasta donde se tiene conocimiento, no hay información disponible sobre la cantidad de GAR producidas en ninguna ciudad de México.

La alternativa de utilizar los aceites de cocina residuales de hogares y de la industria restaurantera es de gran importancia. Sin embargo, un inconveniente del uso de aceites residuales como materia prima para la producción de biodiésel es que tienen propiedades diferentes a las de los aceites refinados o crudos, ya que son degradados por reacciones hidrolíticas y oxidativas que ocurren durante su uso (Costa Neto et al. 2000). Las propiedades de los aceites vegetales residuales dependen del tipo de tratamiento al que son sometidos. El tipo de aceite, duración de la cocción, temperatura del aceite, exposición al aire, periodo de almacenamiento y el tipo de alimento cocinado, determinan la presencia de contaminantes primarios y secundarios, que le darán al aceite residual sus características finales (Encinar et al. 2005, Kulkarni y Dalai 2006, WyseMason y Beckles 2012). Las altas temperaturas de los procesos típicos de cocción y el agua de los alimentos, aceleran la hidrólisis de los triglicéridos e incrementan el contenido de ácidos grasos libres (AGL), impartiendo al aceite un color más oscuro y un olor desagradable (Felizardo et al. 2006).

Por otro lado, los contenidos de agua y de AGL de la materia prima afectan el proceso de transesterificación de glicéridos con alcoholes e interfieren en la separación de los ésteres de ácidos grasos y glicerol (Freedman et al. 1984). El agua, proveniente de las grasas y aceites o la formada durante la reacción de saponificación, retarda la reacción de transesterificación a través de reacciones de hidrólisis (reacción 3). Los triglicéridos pueden ser hidrolizados a diglicéridos y formar más AGL (Leung et al. 2010).

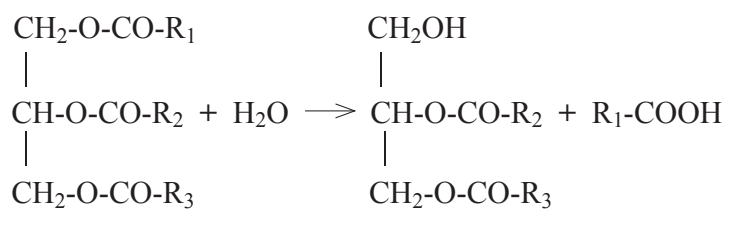

Triglicérido Agua Diglicérido AGL

Una segunda reacción de hidrólisis (reacción 4) puede ocurrir cuando se usa un catalizador alcalino, en la cual los alquil ésteres ya formados (biodieésel) son convertidos en ácidos grasos libres (Abbaszaadeh et al. 2012).

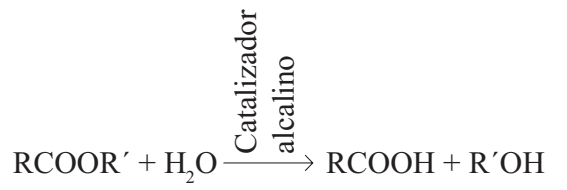

Álquil éster AGL

Por lo tanto, las reacciones secundarias como saponificación e hidrólisis deben ser mantenidas al mínimo durante la transesterificación alcalina.

Lo anterior indica que el éxito del proceso de conversión de grasas y aceites residuales en biodiésel depende de varios factores entre ellos el tipo de catalizador y las características finales de los aceites residuales utilizados como materia prima. Sin embargo, las propiedades fisicas y químicas de las GAR provenientes de restaurantes dependen del tipo de comida y de las prácticas de cocina utilizadas. México tiene una tradición culinaria muy diversa, que se ve reflejada en su amplia variedad de restaurantes. Por lo tanto, la identificación de la calidad de las GAR producidas por los restaurantes en una ciudad del país, es un requisito para establecer condiciones óptimas de operación si se desea establecer un proceso de producción de biodiésel a partir de esta materia prima.

Sobre esta base, el objetivo de este trabajo fue estimar el volumen de grasas y aceites de cocina residuales producidos en la ciudad de Tuxtla Gutiérrez, como un caso de estudio y caracterizarlos para evaluar su potencial como materia prima para la producción de biodiésel. Los resultados obtenidos fueron utilizados para estimar el impacto asociado a la transformación de las GAR provenientes de la industria restaurantera en biodiésel, en relación con el consumo de diésel fósil y en consecuencia, en la reducción de las emisiones de $\mathrm{CO}_{2}$ en México.

\section{MATERIALES Y MÉTODOS}

\section{Materiales}

Todos los reactivos y disolventes utilizados en esta investigación fueron grado analítico. El etanol y el hidróxido de potasio fueron marca Fermont (Productos Químicos Monterrey, Monterey, Nuevo León, México), el tolueno y el hexano, Sigma Aldrich (Sigma-Aldrich, Inc., San Luis, EUA) y el ácido clorhídrico, sulfúrico y metanol fueron J.T. Baker (Mallindckrodt Maker, Inc. Phillipsburg, EUA).

\section{Estimación de la producción de grasas y aceites de cocina residuales (GAR)}

La estimación de la cantidad de grasas y aceites de cocina residuales (GAR) producida en la ciudad 
de Tuxtla Gutiérrez fue realizada en dos etapas. En primer lugar, se contabilizaron los restaurantes registrados en la ciudad y se clasificaron de acuerdo con su tamaño y tipo de cocina. Esto se llevó a cabo entre septiembre y octubre de 2013 y se realizó dividiendo la ciudad en 25 sectores. Cada sector se asignó a un grupo de 6 a 8 personas. Posteriormente, se realizó un monitoreo aleatorio de una vez por semana durante un mes (febrero 2014) a tres restaurantes de cada tipo (previamente clasificados) para estimar la cantidad total de GAR producida en la ciudad por semana. Esto permitió determinar la contribución porcentual de cada tipo de restaurante al volumen total de GAR producido en la ciudad.

\section{Definición y preparación de las muestras de GAR}

Las muestras de GAR recolectadas en los tres restaurantes de cada tipo se mezclaron para conformar una muestra representativa del tipo de cocina. Posteriormente, se preparó una mezcla de GAR (MGAR) de muestras representativas de los diferentes tipos de restaurantes tomando como base la contribución proporcional $(\% \mathrm{v} / \mathrm{v})$ de cada tipo de restaurante al volumen total de GAR producido en la ciudad. Se analizó también una muestra de aceite comercial fresco.

La preparación de las muestras consistió inicialmente en la remoción física de sedimentos y partículas de alimento residuales. Estas fueron calentadas a aproximadamente $40{ }^{\circ} \mathrm{C}$ y se dejaron reposar por dos horas para favorecer la sedimentación. Se cernieron a través de un filtro comercial de $100 \%$ polipropileno, se colocaron en frascos de vidrio color ámbar protegidos de la luz y se almacenaron a $4{ }^{\circ} \mathrm{C}$ hasta su uso.

\section{Caracterización física y química de las GAR}

Las propiedades físicas y químicas de las muestras de GAR se evaluaron usando los métodos de la Asociación Oficial de Químicos Analíticos (AOAC, por sus siglas en inglés). El contenido de ácidos grasos libres se expresó como porcentaje de ácido oleico y se determinó el índice de acidez (método 940.28), índice de saponificación (método 920.160) y contenido de humedad (método 926.12) para evaluar la degradación y la viabilidad de las GAR para la producción de biodiésel. Además, se determinó la densidad y viscosidad (viscosímetro Stabinger modelo SVM 3000), la estabilidad oxidativa (873 Biodiésel Rancimat) y la composición de ácidos grasos.

Para determinar el perfil de ácidos grasos, las muestras fueron primero convertidas a sus respectivos metil ésteres. Para preparar los ésteres de ácidos grasos se utilizaron aproximadamente $5 \mathrm{mg}$ de cada muestra, a las que se les agregó $0.2 \mathrm{~mL}$ de tolueno y $0.4 \mathrm{~mL}$ de $\mathrm{H}_{2} \mathrm{SO}_{4}$ al $1 \%$ en metanol. Las muestras se calentaron a $80{ }^{\circ} \mathrm{C}$ durante 30 min y se dejaron enfriar a temperatura ambiente. Los ésteres de ácidos grasos producidos se extrajeron agregando $1 \mathrm{~mL}$ de hexano y $1 \mathrm{~mL}$ de agua. La fase de hexano se dejó evaporar y los ésteres de ácidos grasos fueron reconstituidos nuevamente en hexano para su análisis (Fozo y Quivey 2004).

La composición de los ésteres metílicos se determinó por cromatografía de gases- espectrometría de masas (GC-MS) (Agilent Technologies modelo 5975 inert XL Net Work GC system) a una temperatura de entrada de $250{ }^{\circ} \mathrm{C}$, flujo en la columna: $1 \mathrm{~mL} / \mathrm{min}$, flujo dividido: $100 \mathrm{~mL} / \mathrm{min}$, volumen de inyección: $1 \mathrm{~mL}$, división: 100:1, temperatura inicial en el horno: $60{ }^{\circ} \mathrm{C}$, tiempo de mantenimiento (1): $5 \mathrm{~min}$, rampa (1): $20^{\circ} \mathrm{C} / \mathrm{min}$ hasta $210^{\circ} \mathrm{C}$, tiempo de mantenimiento (2): $0 \mathrm{~min}$, rampa (2): $1{ }^{\circ} \mathrm{C} / \mathrm{min}$ hasta $213^{\circ} \mathrm{C}$, tiempo de mantenimiento (3): $0 \mathrm{~min}$, rampa (3): $20^{\circ} \mathrm{C} / \mathrm{min}$ hasta $225^{\circ} \mathrm{C}$, tiempo de mantenimiento (4): $25 \mathrm{~min}$, temperatura final del horno: $225^{\circ} \mathrm{C}$, columna: capilar DB-WAX, $60 \mathrm{~m} \times 250 \mathrm{~mm} \times 0.25 \mathrm{~mm}$, gas acarreador: helio.

La composición de los ésteres metílicos en las muestras analizadas se determinó por espectrometría de masas, utilizando el programa de búsqueda NIST Mass spectral NIST/EPA/NIH Mass Spectral (versión de biblioteca 2.0d, 2005).

El índice de saponificación (IS) se estimó de acuerdo con la Ec. 5, la cual relaciona el IS con el índice de acidez (IA) (Canesin et al. 2014):

$$
\text { Éster }(\%)=\frac{I S-I A}{I S} \times 100
$$

\section{RESULTADOS Y DISCUSIÓN}

\section{Clasificación y cuantificación de restaurantes}

Se registraron 793 restaurantes en la ciudad de Tuxtla Gutiérrez y fueron clasificados en ocho categorías con base en el tipo de cocina: 1) restaurantes formales, fueron los establecimientos más grandes donde los platillos se ordenan a partir de un menú, 2) taquerías, donde se sirve todo tipo de tacos, 3 ) rosticerías, donde el pollo rostizado es el platillo principal, 4) cocinas económicas, donde se ofrecen menús con pocos platillos (regionales), bajo el esquema de comida corrida de tres tiempos, 5) gorditas, donde el platillo principal son las gorditas hechas a base de maíz y rellenas principalmente de carne, 
6) bares, donde se sirven botanas, 7) establecimientos de comida rápida, que preparan principalmente: pizza, hamburguesas, papas y pollo frito y 8) antojitos, donde se preparan comidas típicas mexicanas, comúnmente a base de maíz y con un alto contenido de grasas. De éstos, los más numerosos fueron los restaurantes formales, taquerías y cocinas económicas, con $180(23 \%), 167(21 \%)$ y $165(21 \%)$ establecimientos, respectivamente (Fig. 1). Las prácticas de cocina, el tipo de alimento, los aditivos empleados, el aceite utilizado y las veces que el aceite es reutilizado varía en cada restaurante, por lo que la cantidad y características de las GAR producidas por cada tipo de restaurante, también fueron diferentes.

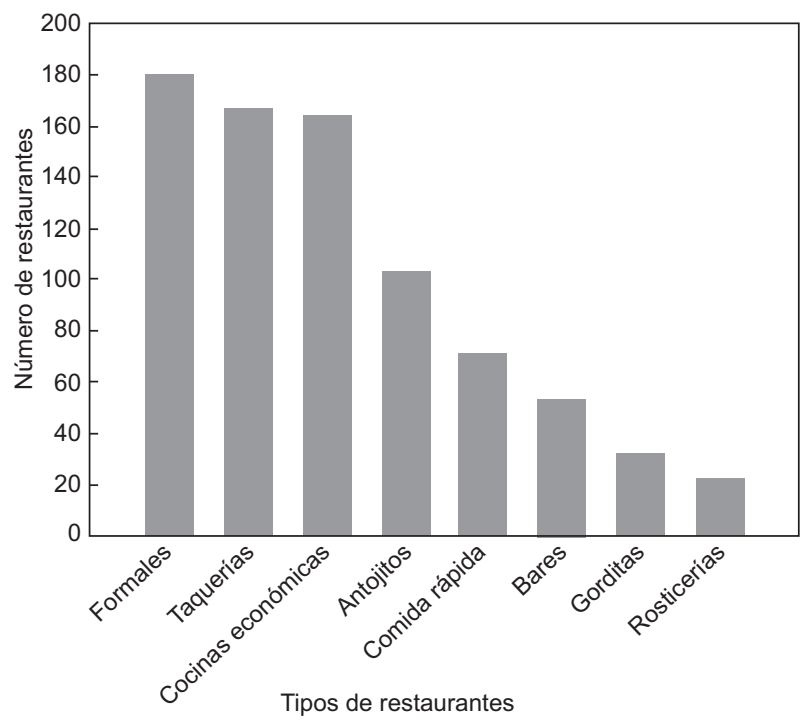

Fig 1. Número y clasificación de restaurantes que fueron registrados en la ciudad de Tuxtla Gutiérrez, Chiapas, México

\section{Estimación de la producción de GAR}

El monitoreo realizado permitió estimar la cantidad total de GAR producida en la ciudad e identificar a los restaurantes que generaron el mayor volumen de éstos. Puede observarse, en la figura 2, que los restaurantes formales, además de ser los más numerosos, fueron los principales generadores de GAR con $1654 \mathrm{~L} /$ semana, así como los restaurantes de comida rápida, los cuales produjeron $963 \mathrm{~L} /$ semana de GAR. Los demás restaurantes tuvieron una producción de GAR similar, la cual varió entre 150 y $298 \mathrm{~L} /$ semana. La producción total semanal de GAR en la ciudad de Tuxtla Gutiérrez fue de 3990 L (Cuadro I). La contribución a la producción total de GAR de los restaurantes formales, comida rápida, taquerías, antojitos, cocinas económicas, bares,

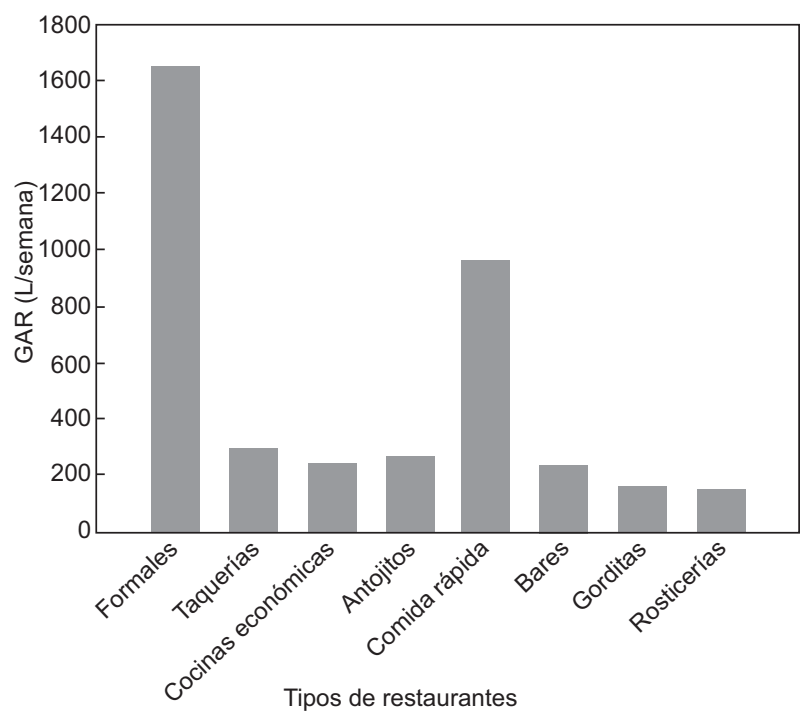

Fig. 2. Volumen de grasas y aceites residuales (GAR) generados por la industria restaurantera en la ciudad de Tuxtla Gutiérrez, Chiapas, México

gorditas y rosticerías fueron de $41 \%, 24 \%, 8 \%$, $7 \%, 6 \%, 6 \%, 4 \%$ y $4 \%$, respectivamente. Tomando como base estas cifras, el volumen total estimado de GAR producido en la ciudad fue de $191524 \mathrm{~L} / \mathrm{año}$, los cuales asumiendo una densidad de $0.9079 \mathrm{~kg} / \mathrm{L}$ equivalen a $174 \mathrm{t}$ de GAR por año.

La población total en la ciudad de Tuxtla Gutiérrez en el año 2010 fue de 553374 habitantes (INEGI 2014), lo que sugiere que los restaurantes producen un promedio de 0.31 t/año de GAR por cada 1000 habitantes. Al considerar esta cifra como una tasa representativa de producción de GAR en México, la cantidad estimada de GAR generada por año sería de 35 kt (38879989 L) en todo el país (112336538 habitantes, INEGI 2014). Cabe señalar que esta es una proyección moderada para el país, ya que la cantidad total de GAR/año generada en ciudades más grandes, como Monterrey o la ciudad de México, podría ser mayor puesto que las personas que viven en ciudades más grandes e industrializadas, comen con mayor frecuencia en restaurantes que las que viven en ciudades pequeñas. La cantidad total de GAR estimada para México es muy baja en comparación con las $113000 \mathrm{kt}$ de GAR de restaurantes y establecimientos de comida rápida que son recolectadas anualmente en los Estados Unidos (Canakci 2007). Sin embargo, es mayor comparada con los 2.5 millones de L/año de GAR generada por los restaurantes en Croacia (Ćosić et al. 2010). Se sabe que la producción de GAR depende de la población y que está relacionada con los niveles de actividad de los sectores restaurantero y hospitalario, los cuales, 
CUADRO I. PROPIEDADES FÍSICAS Y QUÍMICAS DE LOS ACEITES Y GRASAS RESIDUALES Y LA MEZCLA DE ESTOS (MWCOF) OBTENIDOS DE LOS DIFERENTES TIPOS DE RESTAURANTES REGISTRADOS EN LA CIUDAD DE TUXTLA GUTIÉRREZ, CHIAPAS, MÉXICO

\begin{tabular}{lccccccc}
\hline Restaurantes & $\begin{array}{c}\text { Acidez } \\
(\mathrm{mg} \mathrm{KOH} / \mathrm{g})\end{array}$ & $\begin{array}{c}\text { Ácidos grasos } \\
\text { libres }(\%)\end{array}$ & $\begin{array}{c}\text { Saponificación } \\
(\mathrm{mg} \mathrm{KOH} / \mathrm{g})\end{array}$ & $\begin{array}{c}\text { Ester }(\%) \\
\text { Humedad } \\
(\%)\end{array}$ & $\begin{array}{c}\text { Estabilidad } \\
\text { oxidativa }(\mathrm{h})\end{array}$ & $\begin{array}{c}\text { Viscosidad } \\
(\mathrm{mPa} / \mathrm{s})\end{array}$ \\
\hline Antojitos & $0.45(0.01)$ & $0.22(0.01)$ & $185.62(1.76)$ & 99.76 & $0.02(0.01)$ & $2.78(0.06)$ & 37.869 \\
Bares & $1.00(0.01)$ & $0.50(0.01)$ & $193.06(2.33)$ & 99.48 & $0.03(0.02)$ & $5.69(0.07)$ & 39.611 \\
Cocinas & $0.81(0.01)$ & $0.40(0.01)$ & $183.75(1.66)$ & 99.56 & $0.02(0.00)$ & $4.74(0.09)$ & 31.763 \\
Comida rápida & $4.98(0.15)$ & $2.51(0.08)$ & $195.07(4.33)$ & 97.44 & $0.01(0.00)$ & $2.78(0.07)$ & 39.727 \\
Gorditas & $0.94(0.01)$ & $0.47(0.01)$ & $196.31(0.27)$ & 99.52 & $0.01(0.00)$ & $0.86(0.03)$ & 50.027 \\
Formal & $1.62(0.01)$ & $0.81(0.01)$ & $194.66(2.04)$ & 99.17 & $0.02(0.00)$ & $3.89(0.02)$ & 52.205 \\
Rosticerías & $0.99(0.14)$ & $0.50(0.07)$ & $185.55(4.95)$ & 99.46 & $0.03(0.01)$ & $0.44(0.04)$ & 34.104 \\
Taquerías & $1.89(0.14)$ & $0.95(0.07)$ & $192.53(1.29)$ & 99.02 & $0.05(0.00)$ & $2.94(0.04)$ & 36.949 \\
MWCOF & $2.09(0.09)$ & $1.05(0.04)$ & $198.54(0.71)$ & 98.95 & $0.04(0.00)$ & $3.29(0.04)$ & 40.098 \\
Aceite puro & $0.10(0.00)$ & $0.05(0.00)$ & $196.23(1.65)$ & 99.95 & $0.04(0.02)$ & $7.56(0.30)$ & 29.932 \\
\hline
\end{tabular}

a su vez, están vinculados con la actividad general de la economía y en particular con el sector turismo, que se relaciona con el grado de industrialización de cada país (Singh et al. 2010).

\section{Caracterización física y química de las GAR para producción de biodiésel}

Se realizó la caracterización física y química de muestras representativas de GAR provenientes de los diferentes tipos de restaurantes para evaluar su potencial como materia prima para producir biodiésel. Se encontró que el índice de acidez (IA), los niveles de ácidos grasos libres (AGL), la estabilidad oxidativa, la viscosidad y la composición de ácidos grasos, fueron diferentes en todas las muestras de GAR analizadas (Cuadros I y II). Esto era de esperarse ya que todos los restaurantes utilizan diferentes tipos de aceites y la duración de cocción, el tipo de alimento cocinado, los aditivos empleados y las prácticas de cocina son también distintas. Esto mismo ha sido registrado por otros investigadores como WyseMason y Beckles (2012).

Además, se observó que el IA y los niveles AGL de todas las muestras incluyendo la MGAR fueron superiores a los obtenidos con el aceite fresco y que estos índices en las GAR de los restaurantes de comida rápida fueron el doble que los valores determinados en el resto de las GAR analizadas. Esto puede deberse a que la comida rápida es principalmente frita y durante la fritura los niveles de AGL se incrementan como consecuencia de la hidrólisis de los triglicéridos en presencia de humedad y oxidación del alimento (Canakci 2007).

Por el contrario, el porcentaje de humedad y el índice de saponificación presentaron pequeñas variaciones entre las muestras de GAR provenientes de los ocho diferentes tipos de restaurantes y fueron similares a los reportados por Wyse-Mason y Beckles (2012). Estos autores compararon las propiedades de GAR en 11 restaurantes y concluyeron que los valores

CUADRO II. COMPOSICIÓN DE ÁCIDOS GRASOS DE LAS GRASAS Y ACEITES RESIDUALES PROVENIENTES DE LOS DIFERENTES RESTAURANTES ASÍ COMO LA MEZCLA DE ELLOS (MWCOF) Y ACEITE COMERCIAL

\begin{tabular}{lccccccccccc}
\hline Restaurantes & \multicolumn{9}{c}{ Contenido de ácidos grasos (\%) } & \multicolumn{3}{c}{$\begin{array}{c}\text { Saturación } \\
(\%)\end{array}$} & $\begin{array}{c}\text { Insaturación } \\
(\%)\end{array}$ \\
\cline { 2 - 9 } & C14:0 & C16:0 & C16:1 & C18:0 & C18:1 & C18:2 & C18:3 & C20:0 & C20:1 & & \\
\hline Antojitos & 0.54 & 12.13 & 1.10 & 4.45 & 37.62 & 36.51 & 6.58 & 0.47 & 0.60 & $17.58(0.25)$ & $82.42(0.25)$ \\
Bares & 0.34 & 10.89 & 0.80 & 4.40 & 48.59 & 27.46 & 6.46 & 0.30 & 0.76 & $15.93(0.17)$ & $84.07(0.17)$ \\
Cocinas & 0.45 & 10.72 & 0.59 & 3.90 & 40.18 & 35.89 & 7.78 & - & 0.48 & $15.07(0.14)$ & $84.93(0.14)$ \\
Comida rápida & 0.53 & 20.21 & 0.32 & 4.55 & 40.26 & 28.99 & 4.64 & 0.50 & - & $25.79(0.51)$ & $74.21(0.51)$ \\
Gorditas & 0.26 & 10.29 & 0.21 & 4.38 & 46.42 & 30.85 & 6.20 & 0.56 & 0.82 & $15.49(0.29)$ & $84.51(0.29)$ \\
Formales & 0.46 & 17.92 & 0.20 & 4.45 & 38.87 & 32.41 & 5.00 & 0.44 & 0.25 & $23.26(0.21)$ & $76.74(0.21)$ \\
Rosticerías & 1.07 & 24.49 & 5.68 & 6.75 & 38.87 & 21.23 & 1.92 & - & - & $32.30(0.31)$ & $67.70(0.31)$ \\
Taquerías & 2.52 & 27.16 & 3.10 & 15.33 & 40.39 & 10.25 & 0.86 & - & 0.40 & $45.01(0.24)$ & $54.99(0.24)$ \\
MWCOF & 0.62 & 17.82 & 0.51 & 5.75 & 40.98 & 28.77 & 4.51 & 0.50 & 0.55 & $24.69(0.39)$ & $75.31(0.39)$ \\
Aceite puro & - & 8.75 & 0.10 & 3.43 & 43.63 & 34.99 & 7.74 & 0.54 & 0.83 & $12.72(0.13)$ & $87.28(0.13)$ \\
\hline
\end{tabular}


superiores de IA y niveles de AGL obtenidos en las GAR provenientes de los restaurantes de comida rápida fueron resultado de una cocción intensa por periodos de tiempo más largos. Por lo tanto, puede suponerse que las GAR de los restaurantes de comida rápida de la ciudad de Tuxtla Gutiérrez tuvieron una intensidad y duración de cocción superior a la de las GAR de los restaurantes de antojitos, bares, cocinas económicas, gorditas, restaurantes formales, taquerías y rosticerías. Es interesante señalar que el IA y los niveles de AGL reportados por Wyse-Mason y Beckles (2012) para las GAR de los restaurantes de comida rápida y pizzerías fueron más elevados que los determinados en las GAR de esta misma clase de restaurantes en la ciudad de Tuxtla Gutiérrez.

Se observó también una menor estabilidad oxidativa en las muestras de GAR (0.44-5.69 h) de todos los restaurantes respecto a la del aceite fresco $(7.56 \mathrm{~h})$. Este efecto ha sido también relacionado con las reacciones de degradación, tales como autooxidación, polimerización y oxidación térmica, e hidrólisis ocurridas durante la fritura, donde el aceite es sometido repetidamente a altas temperaturas en presencia de aire y humedad (Çaylı y Küsefoğlu 2008).

El IA y el contenido de AGL en el aceite son parámetros clave que determinan la factibilidad de una materia prima para la producción de biodiésel, ya que el rendimiento de la transesterificación alcalina (proceso más utilizado para la producción de biodiésel) es altamente dependiente de la pureza de los reactivos. Los AGL pueden reaccionar con el catalizador alcalino para producir jabón y agua, induciendo la formación de emulsiones. La presencia de agua puede también ocasionar la saponificación del éster a $\mathrm{pH}$ alcalino. Estas reacciones incrementan la cantidad de catalizador requerida para llevar a cabo la transesterificación alcalina y dificultan los procesos de recuperación y purificación del biodiésel (Encinar et al. 2005). Se ha señalado que la materia prima para la producción alcalina de biodiésel debe ser anhidra (contenido de agua $<0.3 \%$ ) y tener un IA inferior a $2 \mathrm{mg} \mathrm{KOH} / \mathrm{g}$ (Freedman et al. 1984, Liu 1994, Canakci 2007). Otros autores han observado que un contenido de AGL cercano a $1 \%(2 \mathrm{mg} \mathrm{KOH} / \mathrm{g})$ en el aceite de cocina residual no tiene efecto en la conversión de ésteres metílicos por transesterificación alcalina (Sharma 2008, Singh 2008, Upadhyay 2008). Con base en lo anterior, las GAR obtenidas de todos los restaurantes monitoreados en la ciudad, excepto las GAR provenientes de restaurantes de comida rápida, podrían ser materia prima adecuada para la transesterificación alcalina, ya que su contenido de AGL fue inferior al $1 \%$ (Cuadro I). Sin embargo, la mezcla de las grasas y aceites de cocina residuales (MGAR) provenientes de todos de restaurantes, incluyendo los de comida rápida, presentó un IA y un nivel de AGL muy cercano a los valores recomendados para la catálisis alcalina. Esto indica que la MGAR es adecuada para la producción de biodiésel y sería una opción para el aprovechamiento de todas las GAR producidas en la ciudad.

Puede verse también en el cuadro I que el contenido de humedad fue similar en todas las muestras e inferior, no sólo al del aceite fresco, sino también a los valores sugeridos en la literatura para la transesterificación alcalina (Encinar et al. 2005, WyseMason y Beckles 2012). Esto puede ser atribuído a que las temperaturas usadas durante la cocción fueron superiores al punto de ebullición del agua, lo que promovió la evaporación de la misma. Por otro lado, los valores de IS indican que la formación potencial de ésteres de todos los tipos de GAR, así como los de su mezcla, sería inferior que la estimada para el aceite fresco (Cuadro I). Sin embargo, resultaría suficiente para obtener altos rendimientos en la producción de biodiésel (98.95\% de ésteres), ya que la Norma EN 14214 recomienda un valor mínimo de $96.5 \%$ de ésteres.

Los valores de viscosidad determinados para todas las GAR recolectadas en la ciudad, especialmente las obtenidas de los restaurantes formales y de gorditas, fueron superiores que los del aceite fresco. Esto puede tener un impacto negativo en el rendimiento del proceso de producción de biodiésel ya que a mayor viscosidad se dificulta el contacto entre las moléculas de aceite y de metanol, lo que disminuye la solubilidad del aceite en el solvente, provocando baja conversión de los triglicéridos (Leung y Guo 2006). Los incrementos en la viscosidad de las GAR podrían atribuirse al complejo patrón de reacciones termolíticas y oxidativas que ocurren en las grasas y aceites durante el proceso de freído. Una alta viscosidad indica la formación de polímeros así como una mayor cantidad y tipos de ácidos grasos presentes en cada muestra (Maskan y Bağc1 2003, Canakci 2007).

\section{Composición de ácidos grasos}

Se determinó la composición de ácidos grasos de las GAR recolectadas en la ciudad, ya que durante la cocción los aceites están expuestos a altas temperaturas en presencia de oxígeno y ésto facilita un importante número de reacciones que alteran la composición de ácidos grasos (Kulkarni y Dalai 2006, Wyse-Mason y Beckles 2012). Los resultados presentados en el cuadro II indican que el ácido oleico (C18:1) se incrementó solamente en las muestras 
provenientes de bares y gorditas. Lo anterior puede deberse a la hidratación de los ácidos grasos más insaturados por la presencia de agua y altas temperaturas durante la cocción (Knothe y Steidley 2009, Wyse-Mason y Beckles 2012). Por el contrario, las muestras de los diferentes tipos de restaurantes presentaron un incremento en ácido palmítico (C16:0) y ácido esteárico (C18:0). Este efecto puede atribuirse a la ruptura de los ácidos grasos insaturados durante la cocción moderada, ya que si bien los ácidos grasos saturados pueden también reaccionar para producir alcanos, alquenos, dióxido de carbono y ácidos grasos de cadena más corta, esto sólo ocurre cuando los aceites son sujetos a condiciones extremas y uso prolongado (Kulkarni et al. 2012). Por lo tanto, la presencia de ácido mirístico (un ácido graso de cadena más corta) en todas las muestras, excepto en la de aceite fresco, y la disminución de los niveles de ácido oleico observados en las GAR de antojitos, cocina económica, comida rápida, restaurantes formales, taquerías y rosticerías, son indicativos de uso prolongado de los aceites en la mayoría de los restaurantes (Cuadro II).

Por el contrario, la menor concentración de ácidos grasos más insaturados (ácido linoleico y linolénico) en todas las muestras de GAR respecto al aceite fresco, señala que los ácidos grasos insaturados son menos estables que los ácidos grasos saturados (Wyse-Mason y Beckles 2012). La concentración menor de ácidos grasos poliinsaturados (linoleico y linolénico) encontrada en las GAR puede ser positiva ya que el biodiésel obtenido a partir de esta materia prima podría tener una mejor estabilidad oxidativa durante el almacenamiento (Canesin et al. 2014).

Asimismo, en el cuadro I puede observarse que la concentración de ácidos grasos saturados (AGS) en todas las muestras de GAR fue mayor (entre 15.07 y $45.01 \%$ ) que la del aceite fresco $(12.72 \%)$. El contenido superior de AGS encontrado en las GAR provenientes de rosticerías $(32.30 \%)$ y taquerías $(45.01 \%)$, puede ser debido al hecho de que ambas muestras son principalmente grasas de origen animal y es sabido que las grasas animales tienen una mayor concentración de ácidos grasos saturados que los aceites vegetales. Esta característica de las GAR provenientes de taquerías y rosticerías es indeseable, ya que las cadenas saturadas pueden causar un incremento en parámetros como la viscosidad, el punto de obstrucción y el punto de nube del biodiésel producido. Sin embargo, los componentes saturados como el ácido mirístico (C14:0), ácido palmítico (C16:0) y ácido esteárico
(C18:0) tienen altos números de cetano y son menos propensos a la oxidación que los componentes insaturados (Canakci 2007). Además, los ácidos grasos saturados, juegan un papel importante en la determinación de las propiedades de flujo en frío del biodiésel, como el punto de obstrucción del filtro en frío (POFF), el cual se produce a altas temperaturas, cuando el contenido de ácidos grasos saturados aumenta. Por ejemplo, si el biodiésel se produce a partir de grasas animales (22-45\% AGS), el POFF puede producirse entre $12{ }^{\circ} \mathrm{C} \mathrm{y}-1{ }^{\circ} \mathrm{C}$ aproximadamente, pero si se deriva de aceite de colza $(5-10 \%$ AGS), el POFF puede ocurrir entre $-7{ }^{\circ} \mathrm{C}$ y $-12{ }^{\circ} \mathrm{C}$, aproximadamente (Chhetri et al. 2008). Para esta propiedad dependiente del clima, los estándares de la EN14214 (2008) reportan para climas templados $6{ }^{\circ}$ de POFF: $5,0,-5,-10,-15,-20^{\circ} \mathrm{C}$.

Basados en el criterio del POFF, las GAR de antojitos, bares, cocinas económicas y gorditas, que tuvieron menor cantidad de AGS (17.58\%, $15.93 \%, 15.07 \%$ y $15.49 \%$, respectivamente), serán una mejor materia prima para la producción de biodiésel que las grasas animales u otros aceites con mayor contenido de ácidos grasos saturados. Por otro lado, las muestras de GAR provenientes de antojitos, bares, cocinas económicas y gorditas presentaron un nivel de ácidos grasos insaturados muy cercano al del aceite fresco. Sin embargo, se determinaron bajos contenidos de ácidos grasos insaturados en las muestras de comida rápida y restaurantes formales. Estos contenidos fueron aún menores en las muestras de GAR provenientes de taquerías y rosticerías (Cuadro II). La presencia de ácidos grasos insaturados en los FAME es necesaria para limitar su solidificación. Sin embargo, los FAME con mayor grado de insaturación no son deseables en el biodiésel, ya que las moléculas insaturadas reaccionan con el oxígeno atmosférico y son convertidas en peróxidos. Además, puede ocurrir entrecruzamiento en el sitio de insaturación y obtenerse un material polimerizado tipo plástico. A altas temperaturas, como las comúnmente encontradas en los motores de combustión interna, el proceso puede acelerarse y el motor rápidamente quedará engomado con los FAME polimerizados (Mohibbe et al. 2005). En este contexto, el biodiésel producido con GAR de taquerías y rosticerías, sería menos susceptible a la degradación que el biodiésel obtenido de GAR de antojitos, bares, cocinas económicas, gorditas, comida rápida y restaurantes formales.

El contenido de ácidos grasos saturados e insaturados de la MGAR fueron superiores a los del 
aceite fresco y similares a las muestras de comida rápida y restaurantes formales, debido a la alta proporción de éstos en la definición de la MGAR. Lo que sugiere que el biodiésel producido al utilizar la MGAR como materia prima, presentará problemas de flujo en frío. Sin embargo, el riesgo disminuye en climas cálidos como el de la ciudad de Tuxtla Gutiérrez.

Uso potencial de las grasas y aceites residuales para producción de biodiésel en México

La cantidad de biodiésel que podría ser obtenida a partir del volumen de GAR producido (174 t/año) en la ciudad de Tuxtla Gutiérrez es de alrededor de 172 t/año, si esta materia prima fuera convertida con una eficiencia del $98.95 \%$ en metil ésteres por transesterificación alcalina (Cuadro I). Sin embargo, considerando un $5 \%$ de incremento en el consumo de combustible cuando se utiliza biodiésel en lugar de diésel (Andersen y Weinbach 2010), las 172 t/año de biodiésel obtenidas a partir de GAR podrían reemplazar 163 t/año de diésel fósil.

Por otro lado, la combustión del diésel fósil resulta en emisiones de $3.52 \mathrm{~kg} \mathrm{CO} / \mathrm{kg}$ diésel (Andersen y Weinbach 2010). Por lo tanto, la sustitución de 163 t/año de diésel fósil producidas a partir de GAR, teóricamente resultaría en una disminución de 575 t/año de las emisiones de $\mathrm{CO}_{2}$. Sin embargo, la recolección, el procesamiento, el consumo de metanol y la distribución asociadas a la producción y uso del biodiésel, implican la combustión de diésel fósil y reducen en un $20 \%$ el impacto en las emisiones de $\mathrm{CO}_{2}$, derivadas del reemplazo de diésel fósil por biodiésel (Andersen y Weinbach 2010). Con base en lo anterior, el proceso de obtención de 172 t/año de biodiésel a partir de GAR generaría por sí mismo $121 \mathrm{t} / \mathrm{año}$ de $\mathrm{CO}_{2}$, por lo que la reducción real en las emisiones de $\mathrm{CO}_{2}$ al aire debido al reemplazo de diésel fósil por biodiésel, serían 454 t de $\mathrm{CO}_{2} /$ año. Esta tendría sin duda un impacto positivo en la reducción de la contaminación en la ciudad.

La cantidad proyectada de GAR producidas por la industria restaurantera en todo México, fue de $35 \mathrm{kt} / \mathrm{año}$. Esta materia prima permitiría producir $34.9 \mathrm{kt} / \mathrm{año}$ de biodiésel (aproximadamente 687 barriles/día), los cuales podrían reemplazar $33 \mathrm{kt} / a n ̃ o$ de diésel fósil y evitar la emisión de $92 \mathrm{kt} \mathrm{CO}_{2} /$ año. El consumo total de diésel en México en el año 2013 fue de aproximadamente $20304 \mathrm{kt}$ (SIE 2015). Por lo tanto, el biodiésel obtenido a partir de GAR podría reemplazar el $0.16 \%$ del diésel utilizado en México y reducir el $0.13 \%$ de las emisiones de $\mathrm{CO}_{2}$ de todo el país. Esto podría parecer una pequeña contribución a las emisiones totales de $\mathrm{CO}_{2}$ en México. Sin embargo, es evidente que esta cifra puede ser superior si las GAR provenientes de otros sectores, así como las grasas animales residuales de los mataderos, aves de corral e industria pesquera fueran utilizadas para producir biodiésel, como se hace en países como Noruega (Andersen y Weinbach 2010). Por otro lado, un impacto ambiental positivo asociado a la reutilización de las GAR sería la disminución de la contaminación del agua y sus repercusiones, ocasionada por las descargas de este residuo cuando no es aprovechado.

\section{CONCLUSIONES}

Se determinaron la producción y las propiedades físicas y químicas de las grasas y aceites residuales provenientes de una variedad de restaurantes en una ciudad de tamaño medio de México. Se identificaron ocho tipos de restaurantes, pero los principales generadores de GAR fueron los restaurantes formales y los de comida rápida. Los resultados indicaron que las GAR provenientes de casi todos los restaurantes, así como la mezcla de éstos, son una materia prima adecuada para la producción de biodiésel. La proyección de estos resultados a todo el país indicó que el procesamiento de las GAR provenientes de la industria restaurantera podría incrementar la producción de biodiésel en México y reducir las emisiones de $\mathrm{CO}_{2}$ generadas por el uso de diésel fósil. Debe señalarse que este estudio es el primero realizado en México en este tema, además, puede contribuir a establecer una política de reúso o conversión de las GAR en biodiésel o en otros productos de valor agregado. También puede ser útil para evaluar el impacto de la descarga de estos residuos al ambiente, así como para promover la producción y uso del biodiésel en México, donde su producción en 2012 fue únicamente de 0.1 miles de barriles/día, mientras que en Estados Unidos se produjeron 64 mil barriles/día en ese mismo año (EIA 2015).

\section{AGRADECIMIENTOS}

Los autores agradecen al Dr. Miguel Ángel García Alvarado por su apoyo en la selección del método usado para llevar a cabo el monitoreo de la producción de grasas y aceites en la ciudad y a Celina Luján Hidalgo por su asistencia técnica en el laboratorio. Veymar G. Tacias agradece también al CONACyT por la beca de doctorado otorgada. 


\section{REFERENCIAS}

Abbaszaadeh A., Ghobadian B., Omidkhah M. R y Najafi G. (2012). Current biodiesel production technologies: A comparative review. Energ. Convers. Manage. 63, 138-148. DOI: 10.1016/j.enconman.2012.02.027.

Andersen O. y Weinbach J.E. (2010). Residual animal fat and fish for biodiesel production. Potentials in Norway. Biomass Bioenerg. 34, 1183-1188.

DOI: 10.1016/j.biombioe.2010.03.010.

AOAC (1990). 926.12 Moisture and volatile matter. Official Methods of Analysis. Association of Official Analytical Chemists Inc. Washington, DC, EUA, 957 pp.

AOAC (1990). 926.160 Saponification number (Koettstorfer Number) of oils and fats. Official Methods of Analysis. Association of Official Analytical Chemists, INC. Washington, DC, EUA, 957 pp.

AOAC (1990). 940.28 Fatty acids (free) in crude and refined oils. Official Methods of Analysis. Association of Official Analytical Chemists, Inc. Washington, DC, EUA, 957pp.

Canakci M. (2007). The potential of restaurant waste lipids as biodiesel feedstocks. Biores. Technol. 98, 183-190. DOI: 10.1016/j.biortech.2005.11.022.

Canesin E. A., de Oliveira C. C., Matsushita M., Dias L. F., Pedrão M. R. y de Souza N. E. (2014). Characterization of residual oils for biodiesel production. Electro. J. Biotechn. 17, 39-45. DOI: 10.1016/j.ejbt.2013.12.007.

Cao P., Tremblay A. Y., Dubé M. A. y Morse K. (2006). Effect of membrane pore size on the performance of a membrane reactor for biodiesel production. Ind. Eng. Chem. Res. 46, 52-58. DOI: 10.1021/ie060555o.

Çaylı G. y Küsefoğlu S. (2008). Increased yields in biodiesel production from used cooking oils by a two step process: Comparison with one step process by using TGA. Fuel Proc. Technol. 89, 118-122.

DOI: 10.1016/j.fuproc.2007.06.020.

Chhetri A. B., Watts K. C. e Islam M. R. (2008). Waste cooking oil as an alternate feedstock for biodiesel production. Energies 1, 3-18. DOI: 10.3390/en1010003.

CEN (2008). EN14214. Automotive fuels- Fatty acid methyl esters (FAME) for diesel engines- Requirements and test methods. European Committee For Standarization. Organización Internacional para la Estandarización (ISO), 25 de octubre, 2008, 15 pp.

Ćosić B., Krajačić G. y Duić N. (2010). Estimation of used cooking oil potential for biofuels production in Republic of Croatia. Memorias. Energy and the Environment 2010. Rijeka, Croacia, 18 al 22 de octubre, 2010. PDF

Costa Neto P. R., Rossi L. F. S., Zagonel G. F. y Ramos L. P. (2000). Produção de biocombustível alternativo ao óleo diésel através da transesterificação de óleo de soja usado em frituras. Quím. Nova 23, 531-537.

Dias J. M., Alvim-Ferraz M. C. M. y Almeida M. F. (2009). Production of biodiesel from acid waste lard. Biores. Technol. 100, 6355-6361.

DOI: 10.1016/j.biortech.2009.07.025.

EIA (2015). International energy statistics: Biodiesel production. U.S. Department of Energy U.S. Energy Information Administration (EIA) [en línea]. http:// www.eia.gov/cfapps/ipdbproject/iedindex $3 . c f m$ ?tid= 79\&pid $=81 \&$ aid $=1 \&$ cid $=$ regions \& syid $=2008 \&$ eyid $=2012$ \&unit $=$ TBPD 09/02/2015.

Encinar J. M., González J. F. y Rodríguez-Reinares A. (2005). Biodiesel from used frying oil. Variables affecting the yields and characteristics of the biodiesel. Ind. Eng. Chem. Res. 44, 5491-5499.

DOI: $10.1021 / \mathrm{ie} 040214 \mathrm{f}$.

Felizardo P., Neiva Correia M. J., Raposo I., Mendes J. F., Berkemeier R. y Bordado J. M. (2006). Production of biodiesel from waste frying oils. Waste Manage. 26, 487-494. DOI: 10.1016/j.wasman.2005.02.025.

Fozo E. M. y Quivey R. G. (2004). Shifts in the membrane fatty acid profile of Streptococcus mutans enhance survival in acidic environments. App. Env. Microbiol. 70, 929-936. DOI: 10.1128/AEM.70.2.929-936.2004.

Freedman B., Pryde E. H. y Mounts T. L. (1984). Variables affecting the yields of fatty esters from transesterified vegetable oils. JAOCS 61, 1638-1643.

DOI: $10.1007 / B F 02541649$.

Huang G., Chen F., Wei D., Zhang X. y Chen G. (2010). Biodiesel production by microalgal biotechnology. Appl. Energ. 87, 38-46.

DOI: 10.1016/j.apenergy.2009.06.016.

Ibeto C., Ofoefule A. y Ezeugwu H. (2011). Fuel quality assessment of biodiesel produced from groundnut oil (Arachis hypogea) and its blend with petroleum diesel. Am. J. Food Technol. 6, 798-803.

DOI: $10.3923 /$ ajft.2011.798.803.

INEGI (2014). Población, hogares y vivienda. Instituto Nacional de Estadística y Geografía [en línea] http://www3.inegi.org.mx/Sistemas/temas/Default. aspx? $\mathrm{s}=\mathrm{est} \& \mathrm{c}=17484 \quad 30 / 11 / 2014$.

Knothe G. y Steidley K. R. (2009). A comparison of used cooking oils: A very heterogeneous feedstock for biodiesel. Bioresource Technol. 100, 5796-5801.

DOI: 10.1016/j.biortech.2008.11.064.

Kulkarni M. G. y Dalai A. K. (2006). Waste cooking oil an economical source for biodiesel: A Review. Ind. Eng. Chem. Res. 45, 2901-2913. DOI: $10.1021 / \mathrm{ie} 0510526$.

Leung D. Y. C. y Guo Y. (2006). Transesterification of neat and used frying oil: Optimization for biodiesel 
production. Fuel Proc. Technol. 87, 883-890. DOI: 10.1016/j.fuproc.2006.06.003.

Leung D. Y. C., Wu X. y Leung M. K. H. (2010). A review on biodiesel production using catalyzed transesterification. Appl. Energ. 87, 1083-1095. DOI: 10.1016/j.apenergy.2009.10.006.

Liu K.S. (1994). Preparation of fatty acid methyl esters for gas-chromatographic analysis of lipids in biological materials. JAOCS 71, 1179-1187.

DOI: $10.1007 / \mathrm{BF} 02540534$.

Maskan M. y Bağc1 H. (2003). Effect of different adsorbents on purification of used sunflower seed oil utilized for frying. Eur. Food Res. Technol. 217, 215-218. DOI: 10.1007/s00217-003-0731-2.

Mohibbe Azam M., Waris A. y Nahar N. M. (2005). Prospects and potential of fatty acid methyl esters of some non-traditional seed oils for use as biodiesel in India. Biomass Bioenerg. 29, 293-302. DOI: 10.1016/j.biombioe.2005.05.001.

Predojević Z. J. (2008). The production of biodiesel from waste frying oils: A comparison of different purification steps. Fuel 87, 3522-3528.

DOI: 10.1016/j.fuel.2008.07.003.
Sharma Y. C., Singh B. y Upadhyay S. N. (2008). Advancements in development and characterization of biodiesel: A review. Fuel 87, 2355-2373.

DOI: 10.1016/j.fuel.2008.01.014.

SIE (2015). IMP: Balance nacional de diésel. Sistema de Información Energética. Secretaría de Energía [en línea]. http://sie.energia.gob.mx/bdiController.do?act ion=cuadro\&subAction=applyOptions 09/02/2015.

Singh A., Smyth B. M. y Murphy J. D. (2010). A biofuel strategy for Ireland with an emphasis on production of biomethane and minimization of land-take. Ren. Sust. Energy Rev. 14, 277-288.

DOI: 10.1016/j.rser.2009.07.004.

Tomasevic A. V. y Siler-Marinkovic S. S. (2003). Methanolysis of used frying oil. Fuel Proc. Technol. 81, 1-6. DOI: 10.1016/S0378-3820(02)00096-6.

Wyse-Mason R. R. y Beckles D. M. (2012). An investigation of restaurant waste oil characteristics for biodiesel production in Trinidad and Tobago. Energy Sust. Dev. 16, 515-519. DOI: 10.1016/j.esd.2012.05.005. 